\title{
Challenging clinical cases in HCV infection
}

\author{
Alessio Strazzulla ${ }^{1 \dagger}$, Giovanni Matera $^{2^{*} \dagger}$, Selma Valerie Mammone ${ }^{1}$, Vittoria Vaccaro ${ }^{1}$, Vincenzo Pisani ${ }^{1}$, \\ Chiara Costa ${ }^{1}$, Francesco Manti ${ }^{3}$, Patrizia Doldo ${ }^{4}$, Lucio Cosco ${ }^{5}$, Francesco Quintieri ${ }^{5}$, Francesco Cesario ${ }^{6}$, \\ Maria Carla Liberto ${ }^{2}$, Aida Giancotti ${ }^{2}$, Carlo Torti ${ }^{1,7}$, Alfredo Focà ${ }^{2}$ \\ From Third Workshop of the HCV Study Group in the Calabria Region \\ Catanzaro, Italy. 04 October 2013
}

\begin{abstract}
We present clinical cases, which underline some difficulties in diagnosis and treatment of hepatitis C virus (HCV) infection. Case report \#1 shows a patient who avoided clinical follow-up for HCV until the development of hepatocellular carcinoma. In this patient, non-invasive procedures did not allow to make a differential diagnosis between hydatidosis and hepatocellular carcinoma but diagnosis was only made with liver biopsy.

In the case report \#2, 24-week treatment with peg-interferon $\alpha 2$ and ribavirin was successfully administered to a HCV genotype-1b infected patient. Shortening HCV treatment did not impair sustained virological response, probably because HCV RNA was low $(<200,000$ IU/I) at baseline.

Lastly, a case series of patients (\#3-6) with hemoglobinopathies is described. Sustained virological response after peg-interferon $\alpha 2$ and ribavirin was achieved in 2 out of 4 patients. While no severe treatment limiting hematological effects were encountered, patients needed more frequent blood transfusions. Thus, new anti-HCV schemes without peg-interferon and ribavirin are urgently needed.
\end{abstract}

\section{Case report \#1 - Hepatocellular carcinoma or echinococcosis? Considerations from a challenging case of neglected HCV}

Alessio Strazzulla, Giovanni Matera, Selma Valerie Mammone, Vittoria Vaccaro, Vincenzo Pisani, Chiara Costa, Francesco Manti, Patrizia Doldo, Maria Carla Liberto, Aida Giancotti, Carlo Torti, Alfredo Focà

\section{Background}

Southern Italy is currently a hyper-endemic area for hepatitis $\mathrm{C}$ virus (HCV) infection. Moreover, it has been shown that incidence of genotype 4 (gt-4) is increasing in the Calabria region [1]. These data, however, do not reflect the actual prevalence of infection, because there is a large proportion of cases that are not diagnosed or not retained into care. For 18 years, it has been reported that chronic HCV infection is one major cause of hepatocellular carcinoma (HCC) [2]. Thus, early diagnosis

\footnotetext{
* Correspondence: gm4106@gmail.com

† Contributed equally

${ }^{2}$ Institute of Microbiology, Department of Health Sciences, "Magna Graecia" University of Catanzaro, Catanzaro, Italy

Full list of author information is available at the end of the article
}

and treatment of $\mathrm{HCV}$ are important to prevent this complication. Also, early diagnosis of HCC is important to better control the disease with a less aggressive therapy than needed if HCC is diagnosed later on.

Echinococcosis is a ubiquitarious, re-emergent zoonosis. Its prevalence is high in several areas of the Mediterranean region, including Southern Italy [3]. In Southern Italy, the major species of medical and public health concern are Echinococcus granulosus and Echinococcus multilocularis, which cause cystic echinococcosis and alveolar echinococcosis, respectively [3]. Hydatid disease predominantly affects liver and lungs. Both cystic echinococcosis and alveolar echinococcosis are serious diseases with a high fatality rate and poor prognosis if managed inappropriately $[4,5]$.

Our purpose is to report a case, showing several clinical features and radiology data, which made differential diagnosis between HCC and liver echinococcosis quite challenging.

\section{Case presentation}

A 68 year old male patient born and living in Catanzaro (Southern Italy) presented with a history of 2-month 
fever, dyspepsia, decreased appetite, weight loss and nicturia. Written informed consent to show the data in the case report was obtained from the patient.

He had been drinking 1 litre of wine daily and smoking 50 cigarettes, but stopped 10 years before. During the initial consultation the patient denied any chronic viral infection, including $\mathrm{HCV}$. He suffered from diabetes and hypertension, so he was on insulin and antihypertensive therapy. On physical examination, hepatomegaly was found (stiff hepatic margin at $3 \mathrm{~cm}$ below the costal rim). Blood exams showed low iron levels (41 $\mu \mathrm{g} / \mathrm{dl})$, high alkaline phosphatase (189 IU/l), moderately raised aspartate aminotransferase (AST) level (49 IU/l) but normal alanine aminotransferase (ALT) level (31 IU/l); pseudo-cholinesterase level was $3230 \mathrm{IU} / \mathrm{l}$, slightly lower than normal values (range 3650-12920 IU/l), bilirubin, albumin and prothrombin time where within normal values. White cell count was 9,040 cells $/ \mathrm{mm}^{3}$ with $1.1 \%$ eosinophils; red cells were $4,610,000 / \mathrm{mm}^{3}$ and hemoglobin was $12.5 \mathrm{~g} / \mathrm{dl}$ (cut off $<14 \mathrm{~g} / \mathrm{dl}$ ), platelets, were normal. Alpha-fetoprotein was mildly elevated at $31.50 \mathrm{ng} / \mathrm{ml}$ (cut off $\leq 8.1$ ). No clinical and hemato-biochemical signs of cirrhosis were found.

Serology for Salmonella spp. (Widal), Rickettsia spp. (Weil Felix) and Leishmania spp. (IgG and IgM) were negative; serology for Brucella spp. showed negative Wright test and borderline IgG at $12 \mathrm{IU} / \mathrm{ml}$ (cut off $\geq$ $12 \mathrm{IU} / \mathrm{ml}$ ). Echinococcus antibody were at borderline levels with a serum titer of 1:80 (cut off $\geq 1: 80$ ). Hepatitis $\mathrm{B}$ markers (HBcAb, HBsAg and HBsAb) were all negative. Investigation of hepatitis $\mathrm{C}$ virus (HCV) markers showed weakly positive HCV-antibodies, HCV-RNA = 131,000 IU/ml), and genotype 1 (gt-1).

The patient, who did not show any previous imaging exam to compare with, underwent abdominal ultrasound which revealed "a voluminous cystic mass of $11 \times 7 \times 9$ centimeters at VII and VIII liver segments in the context of a liver with normal ultrasound structure" and the contrast-enhanced ultrasound control described "centripetal non-homogeneous impregnation of the lesion with slow and delayed wash out, due to the permanence also in the late phase (120") of either hyperechogenicity or hypoechogenicity in each contrast enhanced phase". Neither portal vein expansion nor other signs of portal hypertension were described. This finding suggested a benign lesion and a hydatid cyst (type 1 of Gharby's classification [6]) could not be excluded. Further investigations were therefore recommended.

A computerized tomography (CT) scan was performed, showing that lesion was unmodified in size and describing it as "mixed hypo-isodense with regular borders", adding also the description of "enlarged lymphonodes of approximately $1.5 \mathrm{~cm}$ at the hepatic hilum", and concluding for a "likely abscessual lesion" (Figure 1). However, this

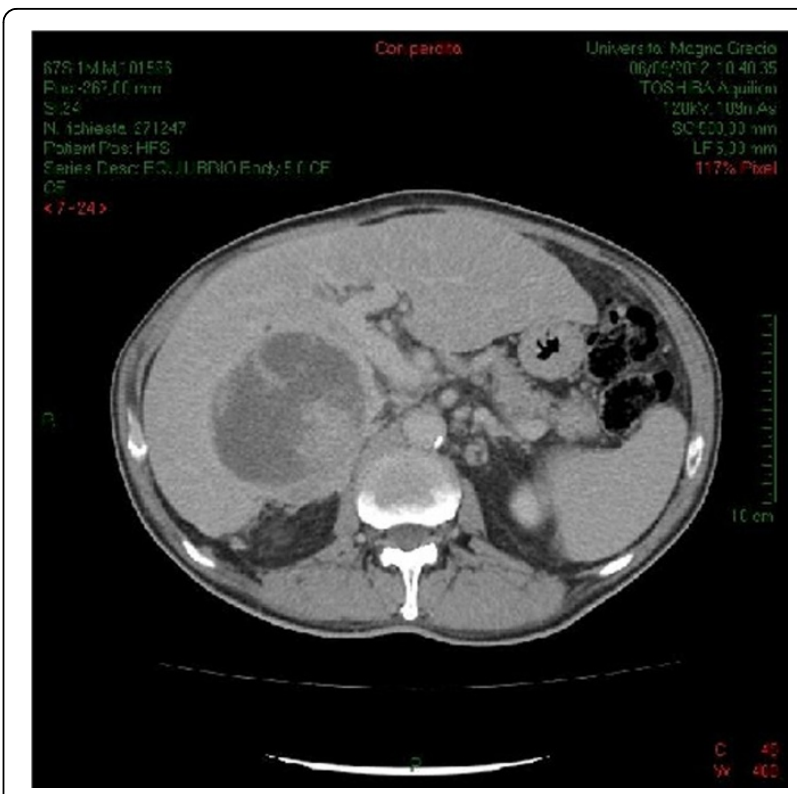

Figure 1 Abdominal contrast enhanced CT scan NOTE: CT scan showed mixed hypo-isodense lesion with regular borders and enlarged lymphonodes of approximately $1.5 \mathrm{~cm}$ at the hepatic hilum.

conclusion did not fit with blood exams, which showed normal white cell count. Moreover, this finding did not fit entirely with the typical presentation of $\mathrm{HCC}$ at $\mathrm{CT}$, which usually appear as hypodense and, only in few cases, presents with a mixed hypo-isodense pattern [7].

Because of inconsistent data, the patient was transferred in a general surgery ward to perform liver biopsy. Due to the suspect of echinococcosis, biopsy was preceded by treatment with albendazole (to avoid peritoneal dissemination of echinococcus scolices). Notably, biopsy proved very difficult to perform, due to the presence of necrotic tissue. Eventually, the histological exam concluded for hepatocellular carcinoma.

\section{Conclusions}

This case highlights the diagnostic difficulties to distinguish HCC from other liver lesions. Furthermore, it shows the importance of an early diagnosis of chronic $\mathrm{HCV}$ in order to prevent severe complications such as liver cirrhosis and HCC.

Serological markers can be useful to make a differential diagnosis between echinococcosis and HCC. However, for echinococcosis, serology can be negative in 10-15\% due to sequestration of echinococcal antigens inside the cyst, especially in case of large lesions $[6,8]$. In our case, the titer of Echinococcus antibodies (1:80) was borderline, not confirming or excluding the suspect of hydatid cyst.

Major current guidelines [9-11] suggest that alphafetoprotein is not diagnostic in absence of a typical 
radiological pattern. Moreover, in our case, alpha-fetoprotein was above the normal range but its values were only mildly elevated $(31.50 \mathrm{ng} / \mathrm{ml})$. However, this finding provided complementary information to imaging, supporting HCC diagnosis especially because the radiological pattern was inconclusive. Clearly, in such a complicated case, neither the CT scan or the haemato-biochemical results (including alpha-fetoprotein) allowed to make the definitive diagnosis that was obtained only through an invasive method (liver biopsy).

In conclusion, this case demonstrates that differential diagnosis between various lesions in the liver may be very complex and requires invasive techniques. It is therefore important to provide a timely diagnosis of late complications and treat them, as soon as possible. Clinical or biochemical features of liver cirrhosis do not necessarily precede $\mathrm{HCC}$, thus they should not be invariably used to select most at risk patients. For these reasons, early diagnosis, HCV treatment, and retention into care are of outstanding importance.

\section{Case report \#2 - Successful treatment of abbreviated Peginterferon/ribavirin in a HCV infected patient with genotype $\mathbf{1 b}$. Could less be more? \\ Francesco Cesario}

\section{Background}

Nowadays, three drug regimens which include pegylated interferon- $\alpha 2$ (PEG-IFN), ribavirin (RBV) and a directly acting antiviral (DAA), namely boceprevir (BOC) and telaprevir (TPR), are the gold standard for the treatment of HCV gt-1 [12,13].

Until 2011, the gold standard was represented by dual therapy, which included PEG-IFN and RBV. At that time, the endorsed guidelines indicated that optimal duration for treatment of gt- 1 and gt- 4 was 48 weeks, while it was 24 weeks for treatment of gt-2 and gt-3 [14].

We present a case of a patient infected by HCV gt-1 who achieved sustained virological response (SVR) with a 24-week PEG-IFN/RBV treatment. Written informed consent to show the data in the case report was obtained from the patient.

\section{Case presentation}

In March 2011, a 45 year old HCV gt-1b positive man was admitted to the Infectious Disease service of the "Annunziata" Hospital of Cosenza (Calabria region) complaining for high AST and ALT blood levels (2-3 folds higher than the upper limit of normality) and depression/anxiety. He was HIV negative, alcohol (>50 gr/day) and drug (heroin and cocaine) addicted until September 2010. Because of his depression/anxiety disorder and his drug addiction history, he was taking citalopram ( $20 \mathrm{mg} /$ day), lorazepam ( $1 \mathrm{mg} / 3$ times per day) and methadone ( $30 \mathrm{mg} /$ day).
On physical examination, hepatomegaly was present (liver protruding 2 centimeters under the rib edge). Blood exams showed elevated AST (170 IU/l), ALT (403 IU/l) and gamma glutamyl transpeptidase (350 IU/l), low albu$\min (1.8 \mathrm{gr} / \mathrm{dl})$ and platelet count $(104.000 \mathrm{cel} / \mu \mathrm{l})$, total and direct bilirubin were 2.0 and $1.2 \mathrm{mg} / \mathrm{dl}$ respectively. Inflammatory markers were raised (erythrocyte sedimentation rate $100 \mathrm{~mm}$, C-reactive protein or $40 \mathrm{mg} / \mathrm{dl}$ ). Ferritin was $540 \mathrm{ng} / \mathrm{ml}$ and hemoglobin was $11 \mathrm{gr} / \mathrm{dl}$. Creatinine level was elevated at $1.8 \mathrm{mg} / \mathrm{dl}$. Metavir score, estimated through liver elastography (Fibroscan) [15], was F4 (13.5 KPa). Overall, Child-Pugh score was 7 (Child A).

The patient underwent abdominal ultrasound, which revealed a slightly enlarged liver and a mass of $5 \mathrm{~cm}$ in the right kidney. Then, an abdominal CT scan described the renal lesion as "likely malignant".

In April 2011, the patient underwent right nephrectomy (Figure 2). A histological exam concluded for granular cell cancer, which is currently considered a malignant renal tumor [16]. Further investigations staged the tumor as T1b-N0-M0, according to the TNM staging system of the Union for International Cancer Control [17].

In May 2011, notwithstanding negative predictors of success (such as alcoholism and liver fibrosis), since HCV-RNA was low $(193,873 \mathrm{IU} / \mathrm{ml})$, patient started a dual therapy with PEG-IFN at a dosage of $1.5 \mu \mathrm{g} / \mathrm{kg}$ per week $(120 \mu \mathrm{g}$ per week) and RBV (1,000 mg/day). A rapid virological response (RVR) with HCV RNA undetectable at week 4 was obtained. Thus, considering RVR, concomitant medications and relevant co-morbidities, a 24-week regimen was preferred rather than the standard 48-week treatment. HCV-RNA was not detectable at week 12 (extended rapid virological response), 24 (end of treatment response) and 6 months after the end of treatment.

\section{Conclusions}

In 2011, HCV guidelines suggested that, in patients infected by gt-1 HCV, duration of treatment could be shortened in case of RVR and low HCV viral load at baseline $(<200,000 \mathrm{IU} / \mathrm{ml})[14,18]$. All these characteristics were present in our case and SVR was actually achieved, notwithstanding the presence of negative features, such as important co-morbidities and a difficult to treat genotype. Interestingly, after achieving RVR, rate of SVR in HCV gt1 patients who received a 24 week long PEG-IFN/RBV treatment was $89 \%$ [18]. This case suggests that treatment duration may vary according to clinical characteristics of patients and type of virological response.

\section{Case series - Treatment of chronic $\mathrm{C}$ hepatitis in patients affected by hemoglobinopathies. Should we really improve?}

Lucio Cosco, Francesco Quintieri 


\section{Background}

Patients affected by hemoglobinopathies, such as thalassemia and sickle cell disease, often develop liver fibrosis due to high iron levels [19]. Moreover, they have a high risk to acquire $\mathrm{HCV}$ via blood transfusions and this occurrence may lead to a worsening of the liver disease [20]. For this reason, treatment of chronic $\mathrm{C}$ hepatitis is important, but it may be less tolerated especially if one considers the risk of anaemia.

\section{Case presentations}

We present a brief case series of patients (Table 1) affected by hemoglobinopathies who underwent treatment with PEG-IFN and RBV at the Infectious Diseases Service of Hospital "Pugliese-Ciaccio" in Catanzaro. Written informed consent to show the data in this case series was obtained from all patients.

a) Case \#3 - In October 2010, a 35 year old woman was admitted to the "Pugliese-Ciaccio" Hospital to start HCV treatment. She was affected by thalassemia major and she knew to be HCV positive since 1990. She had HCV-RNA of 3,590,000 IU/l and gt-2a. ALT was $112 \mathrm{IU} / \mathrm{l}$ and hemoglobin was $10.1 \mathrm{~g} / \mathrm{dl}$. Liver Metavir score, estimated via transient elastography (Fibroscan $^{\mathrm{TM}}$ ) [15], was F2. She started a 24-week treatment with PEG-IFN $2 \mathrm{~b}$ (100 $\mu \mathrm{g}$ per week) and RBV (800 mg/day). RBV dosage was maintained until the end of treatment. Next controls showed not detectable HCV-RNA at weeks 4, 12 and 24. ALT decreased to $61 \mathrm{IU} / \mathrm{ml}$ by week 4 , while it was normal at week 24. After 2 years, HCV-RNA was still not detectable (SVR) and ALT was normal. The patient was also treated with blood transfusions, which increased from a transfusion/month before HCV treatment to a transfusion/10 days during PEG-IFN/RBV therapy. No hemolytic crisis occurred.

b) Case \#4 - In November 2011, a 38 year old, HCV positive and thalassemic (thalassemia major) man started therapy with PEG-IFN 2 2a (180 $\mu$ g per week) and RBV $(800 \mathrm{mg} /$ day). His history of HCV had started in 1991 . He was infected by HCV gt- 4 and, at baseline, HCV-RNA was 18,900,000 IU/l. Moderate fibrosis resulted from liver elastography $(7.9 \mathrm{KPa})$ with an estimated Metavir Score of F2 [15]. Blood exams showed anaemia (hemoglobin $8.9 \mathrm{~g} / \mathrm{dl}$ ). The scheduled duration of treatment was 48 weeks but, unfortunately, treatment was stopped at week 24 because of non response. Indeed, HCV-RNA was detectable at week 4 (3810 IU/l), 12 (3830 IU/l) and 24 (21,900 IU/l). Dosage of RBV was kept at $800 \mathrm{mg} /$ day and no severe hemolysis occurred. After 7 months from the suspension of therapy, viral load was 3,350,000 IU/l. During treatment, the patient received an higher number of blood transfusions (a transfusion/2 weeks) than before treatment (around a transfusion/3 weeks).

c) Case \#5 - In December 2011, a 31 year old man came to the Infectious Diseases Service of Hospital "Pugliese-Ciaccio" to start HCV treatment. He was affected by drepanocytosis (sickle cell disease) and infected by HCV gt -1 b since 1990. At baseline, HCV-RNA was 745,000 IU/l. Mild chronic hepatitis was revealed by liver biopsy (Metavir score F2). ALT was twofold the normal value and hemoglobin was $8.3 \mathrm{~g} / \mathrm{dl}$. He underwent a 18 month long therapy, from December 2011 to June 2013, with PEGIFN 2 2b (100 $\mu$ g per week) and RBV ( $800 \mathrm{mg} /$ day). HCV-RNA was 34,470 IU/l at week 12 and it became not detectable at week 24. At end of treatment HCVRNA was not detectable and ALT was normal. Due to the absence of severe hemolytic crisis, increasing the number of blood transfusions (from $1 / 3$ weeks to $1 / 10$ days ) was the only intervention required to manage anaemia, without any needs to reduce RBV.

d) Case \#6 - In March 2012, a 42 year old woman started PEG-IFN/RBV therapy. She was affected by thalassemia major and she knew to be HCV gt-2a positive since 1991. Blood exams showed anaemia (hemoglobin $8.9 \mathrm{~g} / \mathrm{dl}$ ) and elevated ALT (106 IU/l). The HCV viral load was 3,430,000 IU/l. Liver stiffness (assessed through Fibroscan ${ }^{\mathrm{TM}}$ ) was $8.5 \mathrm{KPa}$ (Metavir score F2) [15]. She followed treatment for 24 weeks with PEG-IFN $\alpha 2 \mathrm{~b}$ (100 $\mu \mathrm{g}$ per week) and RBV (800 mg/day). She reached RVR and end of treatment response, included the normalization of ALT. Unfortunately, 5 months after the end of therapy, she relapsed (HCV-RNA 1,360,000 IU/l). Blood transfusions were performed during treatment more frequently than before therapy (from a transfusion/3 weeks to a transfusion/week). However, reduction of RBV was not necessary and hemolytic crisis did not appear during PEG-IFN/RBV.

\section{Conclusions}

Patients with hemoglobinopathies often exhibit significant liver fibrosis [19]. However, in all of our cases, liver disease, estimated through liver elastography, was moderate (corresponding to F2 at Metavir score). It has to be seen whether elastography is an accurate method to estimate fibrosis in presence of liver damage associated with hemoglobinopathies beside HCV. Interestingly, before HCV treatment, all patients presented with high ALT levels that normalized by the end of treatment. This suggests that, in case of moderate fibrosis, HCV treatment reduces liver inflammation even when SVR is not achieved.

Anaemia is the most common side effect of PEG-IFN/ RBV and patients with hemoglobinopathies require a 


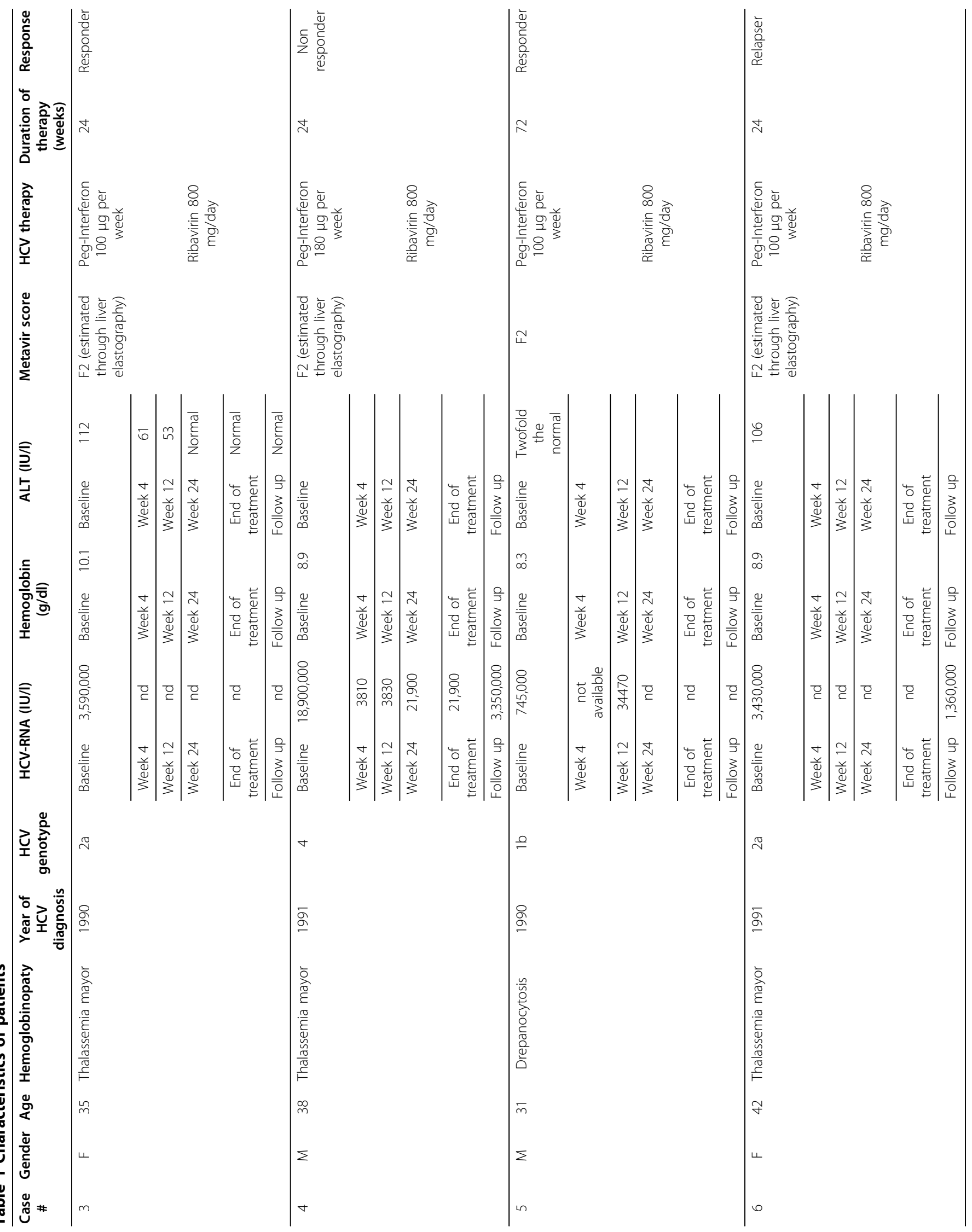


higher number of blood transfusions when they are treated with PEG-IFN/RBV [21]. Indeed, in our short case series, all patients needed more blood transfusions to overcome reduction of hemoglobin. Moreover, anaemia is the most common side effect of DAAs, which represent the current gold standard for treatment of gt-1 HCV in association with PEG-IFN/RBV. Due to the elevated risk of severe anaemia, current available triple therapy with PEG-IFN/ RBV/DAA may be difficult to apply in these patients. Consequently, we are looking forward to the next generation DAAs, which are expected to be strongly effective even in absence of PEG-IFN and RBV.

\section{List of abbreviations}

HCV, hepatitis C virus; gt, genotype; HCC, hepatocellular carcinoma; AST, aspartate aminotransferase; ALT alanine aminotransferase; CEUS, contrastenhanced ultrasound; $\mathrm{CT}$, computerized tomography; HBV, hepatitis B virus; PEG-IFN, pegylated interferon; RBV, ribavirin; DAA, directly acting antiviral; BOC, boceprevir; TPR telaprevir; SVR, sustained viralogical response; RVR, rapid viral response.

\section{Competing interests}

The authors declare that they have no competing interests.

\section{Authors' contributions}

AS contributed to manuscript writing; GM contributed to manuscript writing and manuscript revision; SVM, W contributed to manuscript writing; VP, CC, FM, PD, LC, FQ, FC collected clinical data; MCL contributed to manuscript revision; AG collected laboratory data; $C T$ contributed to manuscript writing and manuscript revision; AF contributed to manuscript revision.

\section{Declarations}

The publication of this supplement was partly supported by an unrestricted grant provided by Roche. The articles were independently prepared by the authors with no input from Roche. Roche were not involved in selecting the articles for the supplement. The pegylated IFN/pegIFN treatment mentioned in this article is produced by Roche.

This article has been published as part of BMC Infectious Diseases Volume 14 Supplement 5, 2014: Proceedings of the Third Workshop of the HCV Study Group in the Calabria Region: Results from the South Italian Network for Rational Guidelines and International Epidemiology (SINERGIE) Project. The full contents of the supplement are available online at http://www. biomedcentral.com/bmcinfectdis/supplements/14/S5.

\section{Authors' details}

'Unit of Infectious Diseases, Department of Medical and Surgical Sciences, University "Magna Graecia", Catanzaro, Italy. "Institute of Microbiology, Department of Health Sciences, "Magna Graecia" University of Catanzaro, Catanzaro, Italy. ${ }^{3}$ Department of Radiology, "Magna Graecia" University of Catanzaro, Catanzaro, Italy. ${ }^{4}$ Centre for Oncology, "Tommaso Campanella" Fundation, Catanzaro, Italy. ${ }^{5}$ Unit of Infectious Diseases, "Pugliese-Ciaccio" Hospital, Catanzaro, Italy. ${ }^{6}$ Unit of Infectious Diseases, "Annunziata" Hospital, Cosenza, Italy. ${ }^{7}$ University Unit of Infectious Diseases, University of Brescia, School of Medicine, Brescia, Italy.

Published: 5 September 2014

\section{References}

1. Liberto MC, Marascio N, Zicca E, Matera G: Epidemiological features and specificities of HCV infection: a hospital-based cohort study in a university medical center of Calabria region. BMC Infect Dis 2012, 12(Suppl 2):S4.

2. Tong MJ, Lee SY, Hwang SJ, Co RL, Lai PPC, Chien D, Kuo G: Evidence for hepatitis $C$ viral infection in patients with primary hepatocellular carcinoma. West J Med 160, 133-138.
3. Moro P, Schantz PM: Echinococcosis: a review. Int I Infect Dis 2009, 13(2):125-133.

4. McManus DP, Zhang W, Li J, Bartley PB: Echinococcosis. Lancet 2003, 362(9392):1295-1304.

5. Dakkak A.: Echinococcosis / hydatidosis: a severe threat in Mediterranean countries. Vet Parasitol 2010, 174:2-11.

6. Nepalia S, Joshi A, Shende A, Sharma SS: Management of echinococcosis. J Assoc Physicians India 2006, 54:458-62, Jun.

7. Szklaruk J, Silverman PM, Charnsangavej C: Imaging in the diagnosis, staging, treatment, and surveillance of hepatocellular carcinoma. AJR Am J Roentgenol 2003, 180(2):441-54, Feb.

8. Nunnari G, Pinzone MR, Gruttadauria S, Celesia BM, Madeddu G, Malaguarnera G, Pavone P, Cappellani A, Cacopardo B: Hepatic echinococcosis: clinical and therapeutic aspects. World I Gastroenterol 2012, 18(13):1448-58, Apr 7.

9. European Association For The Study Of The Liver: European Organisation For Research And Treatment Of Cancer: EASL-EORTC: Clinical practice guidelines: management of hepatocellular carcinoma. J Hepatol 2012, 56:908-943.

10. Bruix J, Sherman M: American Association for the Study of Liver Diseases: Management of hepatocellular carcinoma: an update. Hepatology 2011, 53:1020-1022.

11. Omata M, Lesmana LA, Tateishi R, Chen PJ, Lin SM, Yoshida H, Kudo M, Lee JM, Choi BI, Poon RT, Shiina S, Cheng AL, Jia JD, Obi S, Han KH, Jafri W, Chow P, Lim SG, Chawla YK, Budihusodo U, Gani RA, Lesmana CR, Putranto TA, Liaw YF, Sarin SK: Asian Pacific Association for the Study of the Liver consensus recommendations on hepatocellular carcinoma. Hepatol Int 2010, 4:439-474.

12. Gellad ZF, Reed SD, Muir AJ: Economic evaluation of direct-acting antiviral therapy in chronic hepatitis C. Antivir Ther 2012, 17((6 Pt B)):1189-99.

13. Ghany MG, Nelson DR, Strader DB, Thomas DL, Seeff LB: An update on treatment of genotype 1 chronic hepatitis $C$ virus infection: 2011 practice guideline by the American Association for the Study of Liver Diseases. Hepatology 2011, 54(4):1433-44, Oct.

14. Ghany MG, Strader DB, Thomas DL, Seeff LB: Diagnosis, management and treatment of Hepatitis C: An update. Hepatology 2009, 49(4):1335-74, Apr.

15. Nitta $Y$, Kawabe N, Hashimoto S, Harata M, Komura N, Kobayashi K, Arima Y, Shimazaki H, Nakano T, Murao M, Ichino N, Osakabe K, Aoki H, Hosoe Y, Sugiyama H, Nishikawa T, Yoshioka K: Liver stiffness measured by transient elastography correlates with fibrosis area in liver biopsy in patients with chronic hepatitis C. Hepatol Res 2009, 39(7):675-84, Jul.

16. World Health Organization (WHO) and the International Agency for Research on Cancer (IARC): Classification of Tumours: Pathology and Genetics of Tumours of the Urinary System and Male Genital Organs. IARC Press Lyon 2004.

17. Sobin LH, Gospodarowicz MK, Wittekind C: TNM Classification of Malignant Tumours. 7 edition. Wiley-Blackwell; 2009, 336, November.

18. Jensen DM, Morgan TR, Marcellin P, Pockros PJ, Reddy KR, Hadziyannis SJ, Ferenci $P$, Ackrill AM, Willems B: Early identification of HCV genotype 1 patients responding to 24 weeks peginterferon alpha-2a $(40 \mathrm{kd}) /$ ribavirin therapy. Hepatology 2006, 43(5):954-60, May.

19. Lai ME, Origa R, Danjou F, Leoni GB, Vacquer S, Anni F, Corrias C, Farci $P$, Congiu G, Galanello R: Natural history of hepatitis $C$ in thalassemia major: a long-term prospective study. Eur J Haematol 2013, 90(6):501-7, Jun.

20. Angelucci E, Muretto P, Nicolucci A, Baronciani D, Erer B, Gaziev J, Ripalti M, Sodani P, Tomassoni S, Visani G, Lucarelli G: Effects of iron overload and hepatitis $C$ virus positivity in determining progression of liver fibrosis in thalassemia following bone marrow transplantation. Blood 2002, 100(1):17-21, Jul 1

21. Sood A, Sobti P, Midha V, Singla D, Kaur A, Kaushal S, Sood N, Mittal R, Puri S: Efficacy and safety of pegylated IFN alfa $2 b$ alone or in combination with ribavirin in thalassemia major with chronic hepatitis $C$. Indian J Gastroenterol 2010, 29(2):62-5, Mar.

doi:10.1186/1471-2334-14-S5-S7

Cite this article as: Strazzulla et al:: Challenging clinical cases in HCV infection. BMC Infectious Diseases 2014 14(Suppl 5):S7. 